\title{
Dielectric metasurfaces for distance measurements and three-dimensional imaging
}

\author{
Chunqi Jin, ${ }^{\text {a,b,c }}$ Mina Afsharnia, ${ }^{a}$ René Berlich, ${ }^{d}$ Stefan Fasold, ${ }^{a}$ Chengjun Zou, ${ }^{a}$ Dennis Arslan, ${ }^{a}$ \\ Isabelle Staude, ${ }^{a}$ Thomas Pertsch, ${ }^{\mathrm{a}, \mathrm{d}}$ and Frank Setzpfandt ${ }^{\mathrm{a}, *}$ \\ ${ }^{a}$ Friedrich Schiller University Jena, Institute of Applied Physics, Abbe Center of Photonics, Jena, Germany \\ ${ }^{b}$ Chinese Academy of Sciences, Changchun Institute of Optics, Fine Mechanics and Physics, Changchun, China \\ 'University of the Chinese Academy of Sciences, Beijing, China \\ ${ }^{\mathrm{d}}$ Fraunhofer Institute for Applied Optics and Precision Engineering, Jena, Germany
}

\begin{abstract}
Ultrathin metasurfaces have shown the capability to influence all aspects of light propagation. This has made them promising options for replacing conventional bulky imaging optics while adding advantageous optical properties or functionalities. We demonstrate that such metasurfaces can also be applied for single-lens three-dimensional (3-D) imaging based on a specifically engineered point-spread function (PSF). Using Huygens' metasurfaces with high transmission, we design and realize a phase mask that implements a rotating PSF for 3-D imaging. We experimentally characterize the properties of the realized double-helix PSF, finding that it can uniquely encode object distances within a wide range. Furthermore, we experimentally demonstrate wide-field depth retrieval within a 3-D scene, showing the suitability of metasurfaces to realize optics for 3-D imaging, using just a single camera and lens system.
\end{abstract}

Keywords: optics; nanophotonics; metasurfaces; Mie-resonances; three-dimensional imaging; point-spread function engineering. Received Apr. 14, 2019; accepted for publication Jun. 1, 2019; published online Jun. 18, 2019.

(C) The Authors. Published by SPIE and CLP under a Creative Commons Attribution 4.0 Unported License. Distribution or reproduction of this work in whole or in part requires full attribution of the original publication, including its $\mathrm{DO}$.

[DOI: 10.1117/1.AP.1.3.036001]

\section{Introduction}

Metasurfaces, dense arrangements of subwavelengths scatterers in a plane, have been demonstrated to enable control of transmitted light in both amplitude and phase. ${ }^{1}$ Specifically, metasurfaces manufactured solely from dielectric materials have a large application potential due to their many design degrees of freedom combined with negligible material losses, ${ }^{2-4}$ especially compared to their plasmonic counterparts. ${ }^{5}$ Consequently, a number of different optical elements have been demonstrated, including holograms, ${ }^{6-10}$ vortex-phase plates, ${ }^{11-13}$ lenses, ${ }^{7,14-23}$ and tunable functional elements. ${ }^{24-26}$

In addition to applications in conventional two-dimensional (2-D) imaging, metasurfaces can also be used to replace bulk optics for three-dimensional (3-D) imaging. Numerous approaches for 3-D imaging are in use. ${ }^{27,28}$ Many of these rely on stereoscopic measurements using two cameras, ghostimaging, or compressed-sensing techniques using a series of measurements with a specific illumination. Furthermore, 3-D

*Address all correspondence to Frank Setzpfandt, E-mail: f.setzpfandt @ uni-jena.de images can be measured by using plenoptic cameras, which basically enable measurement of the wavefront in the image plane of an imaging system. Metasurfaces that enable wavefront sensing either by an iterative procedure ${ }^{29}$ or by implementing a lens array like in a Shack-Hartmann sensor ${ }^{30}$ have been already demonstrated. The latter concept, where the metasurface lens array was placed in the image plane of a conventional lens, was used to experimentally implement a metasurface-based plenoptic camera, ${ }^{31}$ albeit only for circularly polarized light. An alternative approach to enable passive single-shot 3-D imaging with just one camera is based on using optical elements with a specifically designed point-spread function (PSF), which unambiguously encodes the object distance. Here, in principle, only one imaging optical element is needed, compared to the separate lens and lens arrays in the plenoptic camera. One particular solution proposed for depth estimation and 3-D imaging ${ }^{32,33}$ employs a PSF with two foci rotating around a central point. This approach is schematically depicted in Fig. 1(a). Light from a point source is focused by an optical element, which consists of a standard lens and an additional phase mask with the phase profile depicted in Fig. 1(b). In the focal plane, this will lead to 
(a)

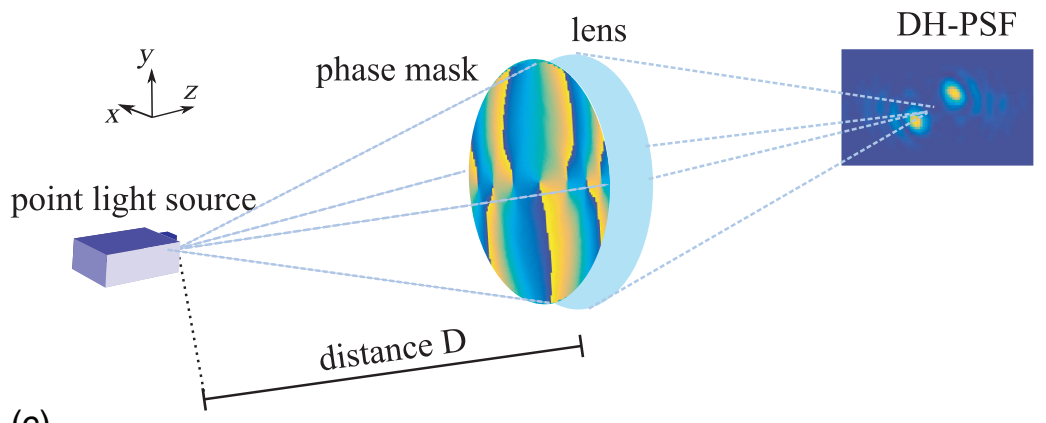

(c)

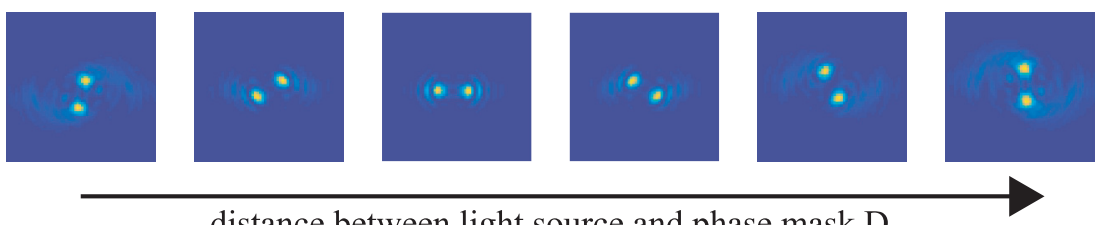

(b)

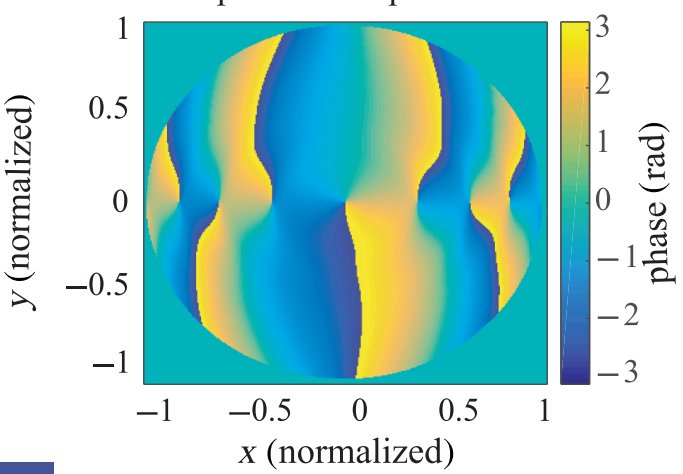

Fig. 1 (a) Scheme showing the creation of a DH-PSF by using a phase mask with the phase profile shown in (b). (c) Intensity distribution in the image plane of the lens for different distances between the point light source and the phase mask.

the creation of two distinct focal spots. Upon movement of the point source with respect to the lens along the optical axis, these spots will rotate around their common center, as shown in Fig. 1(c). Due to the rotation, such double-helix PSFs (DH-PSF) also provide unique information about the direction in which the source is moved, in contrast to a conventional PSF, which spreads symmetrically for both directions of movement. Moreover, it can be shown that a DH-PSF provides superior depth-estimation accuracy over a large depth of focus, which is quantified by the Cramer-Rao lower bound. ${ }^{34}$ This advantageous property has been used for depth localization of fluorescent molecules, allowing the achievement of 3-D fluorescence images. ${ }^{34-36}$ Furthermore, wide-field 3-D imaging using a single camera was demonstrated with an optical element implementing a DH-PSF. ${ }^{37}$ Here, the information from each object point is distributed on two locations in the measured raw image, which are shifted by a fixed distance with respect to each other governed by the DH-PSF. The direction of the shift depends on the distance of the object point from the optical element. The depth map and 2-D image can be obtained from this measurement using numerical postprocessing. ${ }^{37}$

In all works up to now, the phase elements needed to implement DH-PSFs for imaging and depth reconstruction were based on optical elements with varying thickness realized by grayscale lithography ${ }^{37,38}$ or generated using a spatial light modulator (SLM) ${ }^{33}$ The former technology is well established and, after producing one master element, allows for convenient reproduction of optical elements using UV-imprint techniques. ${ }^{39}$ The latter approach using an SLM enables flexible implementation of different DH-PSF designs.

Here, we implement an all-dielectric metasurface realizing a DH-PSF suitable for depth estimation and 3-D imaging, and experimentally demonstrate its functionality by measuring images with depth resolution using a passive, single-lens optical system. Compared to the realization approaches based on classical optical elements, this unlocks more complete control of the spectral and polarization degrees of freedom of the realized PSF, as metasurfaces allow for precise control of spectral and polarization degrees of freedom. Although not in the scope of this work, this control could be used either to reduce the sensitivity of the desired optical functionality on the spectrum or polarization of the incident light, ${ }^{9,12,14,31}$ or on the contrary to enhance and implement specific dispersion- and polarization-dependent functionalities. ${ }^{23,40-42}$ The latter could lead to multimodal 3-D imaging.

\section{Design of the Phase Mask}

Unlike conventional optical elements, metasurfaces use an array of nanoantennas with subwavelength size and separation to achieve optical functionality. Spatially varying geometric parameters of the nanoantennas are used to realize a spatial variation of the optical response, thus shaping the optical wavefront. ${ }^{1}$ To implement the phase mask depicted in Fig. 1(b), we need to realize a spatially varying phase of the transmitted light while ideally maintaining unity transmission. The so-called Huygens' metasurfaces represent one particular way to achieve this. ${ }^{43}$ Here, resonant nanodisk antennas are used, which are designed such that linear scattering is suppressed in the backward direction due to the interference of different multipolar components of the localized resonant fields. In metasurfaces composed from such nanoresonators, all impinging light with a wavelength close to the resonances is transmitted while reflection is prohibited, independent of the polarization. The transmission phase can be controlled by changing the incident optical wavelength with respect to the resonance frequency ${ }^{43}$ or by varying the in-plane geometry of the metasurface, like the size of the nanoresonators or their distance within the metasurface, ${ }^{6,12}$ i.e., the lattice period. In comparison to other possible implementations ${ }^{29,31}$ Huygens' metasurfaces enable realization of particularly thin optical elements better suited for integration with other structures. Furthermore, due to their resonant nature, they feature a strong dispersion, which could be used 
to implement wavelength-dependent functionalities for multimodal sensing.

The phase profile that needs to be implemented by the metasurface can be calculated with arbitrary spatial resolution and previously has been realized by phase masks with continuously changing thickness profiles. However, the optical properties of metasurfaces can only be defined on a spatially discrete grid. The smallest possible spacing is given by the lattice period of the metasurface. Here, we aim to use metasurfaces composed of resonant silicon nanodisks at a wavelength of around $1500 \mathrm{~nm}$, where silicon has negligible absorption losses. Typical periods of Huygens' metasurfaces for this spectral range are around $800 \mathrm{~nm}$, well below the target wavelength. Hence, a quasicontinuous phase profile could, in principle, be realized. However, the local distance of the nanoresonators is an important parameter to control the optical response. Realizing 2-D phase masks with continuously varying distance between the nanoresonators while avoiding stitching errors is challenging, albeit possible. ${ }^{44}$ Here, we instead consider a pixelated metasurface, where each square pixel of $20-\mu \mathrm{m}$ side length contains a locally periodic metasurface with homogeneous optical properties. For a planned phase-mask diameter of $1 \mathrm{~cm}$, this pixel size corresponds to the numerical discretization used to plot the data in Fig. 1(b).

Although, in principle, nanostructures can be manufactured with quasicontinuous changes in their geometrical and optical properties, practically only a limited number of different nanostructures can be explicitly designed. Hence, the used phase values in the phase mask also have to be discretized. To estimate the influence of this discretization on the resulting optical functionality, we first perform numerical simulations. To this end, we consider phase masks discretized using $4,6,8$, and 10 phase levels evenly distributed in the interval from 0 to $2 \pi$. Starting from the reference phase profile shown in Fig. 1(b), the discretization was implemented by replacing every phase value with the nearest of the discrete set of values. Resulting phase functions for 4 and 10 phase levels are shown in Figs. 2(a) and 2(b), respectively.
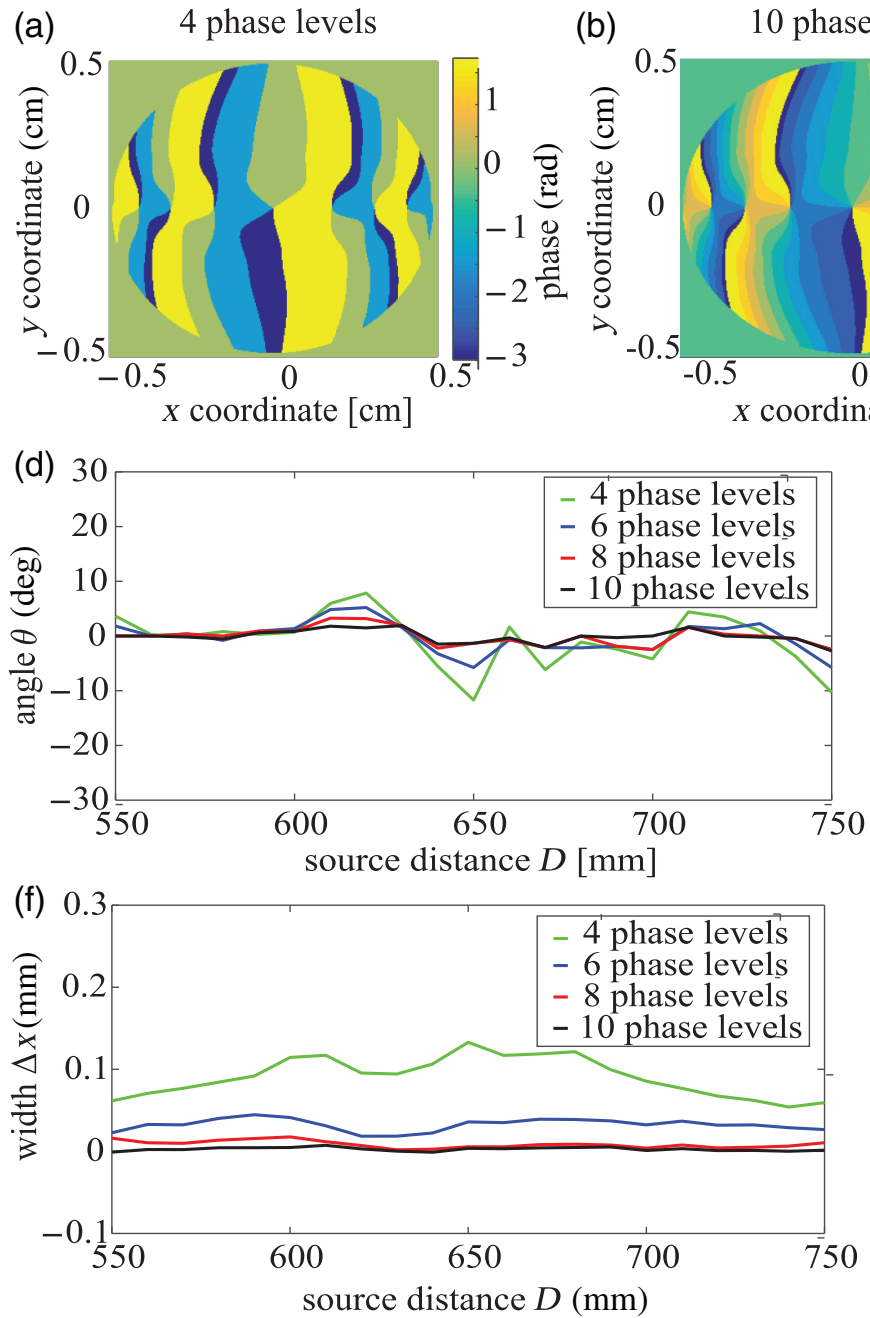

(b) $\quad 10$ phase levels

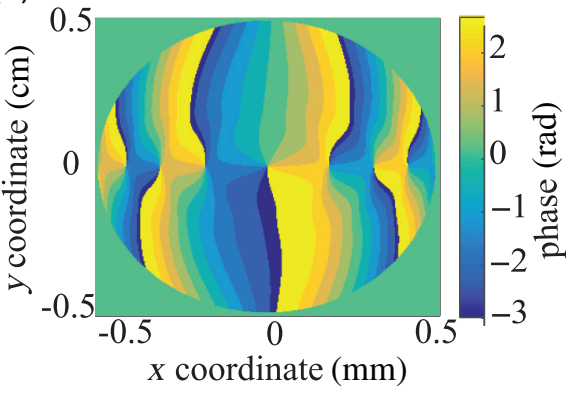

(c)
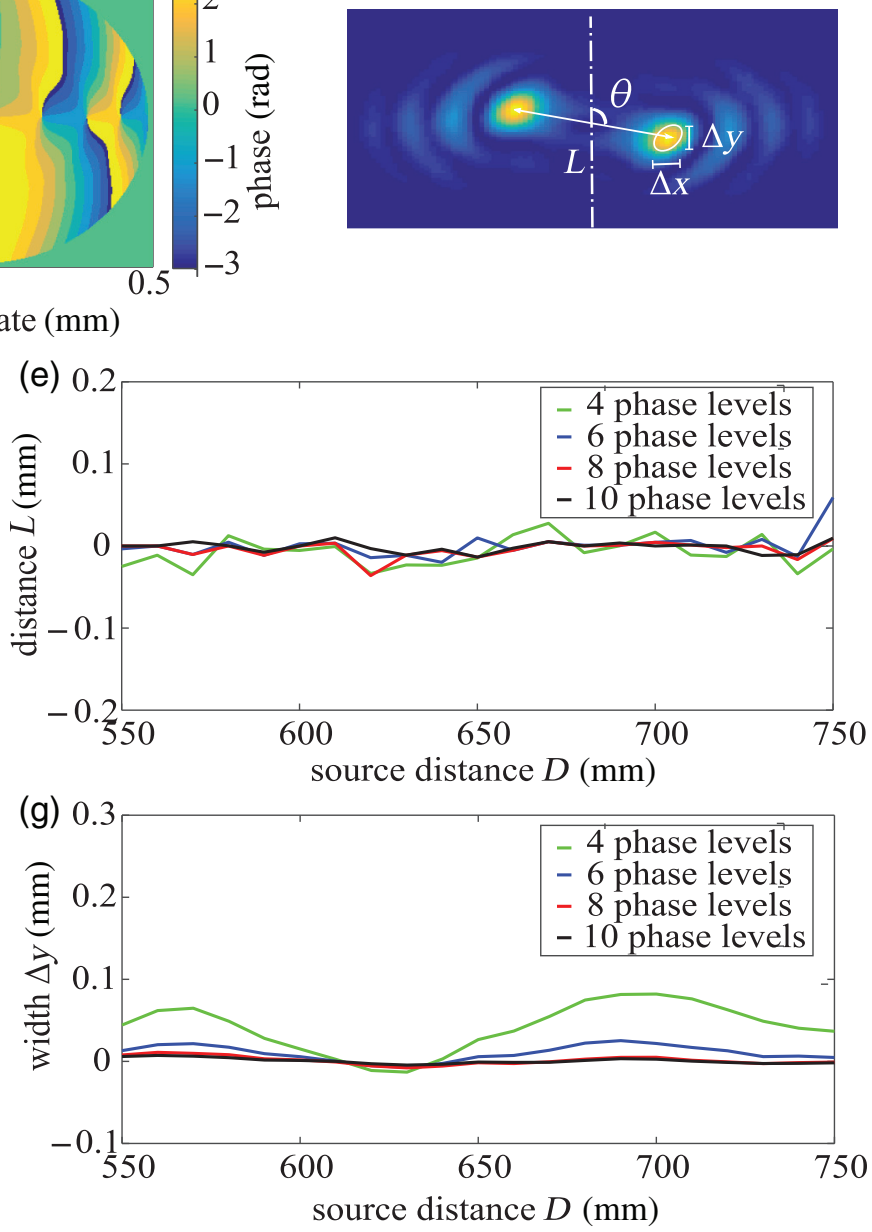

Fig. 2 Phase profiles for realizing a DH-PSF with (a) 4 and (b) 10 evenly spaced phase steps. (c) Intensity profile of ideal DH-PSF, where the main parameters are indicated. (d)-(g) Comparison of parameters of ideal and discretized PSFs dependent on the distance $D$ between the point light source and the phase mask. The plots show the difference between the values for ideal and discretized realizations for $(\mathrm{d})$ the rotation angle $\Theta,(\mathrm{e})$ the distance $L$, (f) the horizontal width $\Delta x$, and (g) the vertical width $\Delta y$. 
To determine the response of this phase mask, we consider an optical setup similar to the one shown in Fig. 1(a). The illuminating beam of $1500-\mathrm{nm}$ wavelength is modeled as a spherical wave originating from a point at a distance $D$ from the phase mask. The phase mask has a diameter of $10 \mathrm{~mm}$; the distances $D$ are around $600 \mathrm{~mm}$. For these parameters, the field resulting from considering a spherical wave is similar to that of a Gaussian beam with a tight focus at the origin of the spherical wave. Directly behind the phase mask, we consider a lens with a focal length of $400 \mathrm{~mm}$, which we model using the thin lens approximation. The intensity distribution is calculated at a distance of $1100 \mathrm{~mm}$ after the lens. One example of a calculated DH-PSF, obtained using the continuous phase mask, is shown in Fig. 2(c). We note that the chosen parameters for the considered optical system are just specific examples. The obtained results concerning the possibility to discretize the phase mask are independent of this specific choice.

To investigate the effect of the phase discretization, we analyze different parameters of the calculated intensity distributions, which are indicated in Fig. 2(c). In particular, these are the rotation angle $\Theta$ of the DH-PSF with respect to the $y$ axis, the distance $L$ between the two maxima, the full-width at half maximum (FWHM) $\Delta x$ of the right lobe along the $x$ direction, and the FWHM $\Delta y$ of the right lobe along the $y$ direction. The parameters $L$ and $\Theta$ were calculated from the simulated intensities by finding the positions of the two maxima and calculating their distance and the inclination of the connecting line. The widths were calculated by fitting Gaussian functions to $x$ - and $y$-cuts through the intensity distribution at the position of the right maximum. For the phase mask without discretization, the values of the parameters are $L=0.17 \mathrm{~mm}$ and $\Delta x=$ $\Delta y=0.05 \mathrm{~mm}$, whereas the angle is changing linearly with the distance of the source $D$. The differences of the values obtained for the discretized phase masks from the ones for the continuous phase mask are plotted in dependence on the source distance $D$ in Figs. 2(d)-2(g). We find that for 6, 8, and 10 phase steps, the differences are small, whereas, for only 4 steps, we find larger deviations in the rotation angle and widths of the individual maxima. From this analysis, we conclude that a discretization with as low as 6 phase steps will not markedly change the geometrical features of the realized DH-PSF. We furthermore analyze the efficiency of the discretized phase masks by calculating the energy concentrated in the two central lobes. For the continuous phase mask, around $30 \%$ of the energy is within the FWHM of the two main lobes. For the discretized phase mask with 10 (6) phase steps, this fraction reduces to $29 \%(24 \%)$. Hence, the performance of the phase mask with respect to 3-D imaging is not expected to change strongly with discretization.

For the implementation of the discretized phase mask, specific nanostructure designs have to be found that implement the required phase values for the transmitted fields. Full $2 \pi$ phase coverage has been achieved with several metasurface designs before by changing in-plane geometry parameters like the size of the nanoresonators or the period of their arrangement. ${ }^{6,9,12,45-48}$

Here, we consider polarization-independent Huygens' metasurfaces consisting of silicon nanodisks in a square lattice. The geometry is schematically shown in Fig. 3(a) together with the (a)

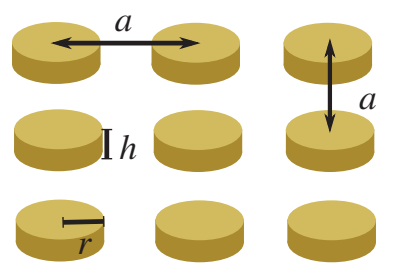

(b)

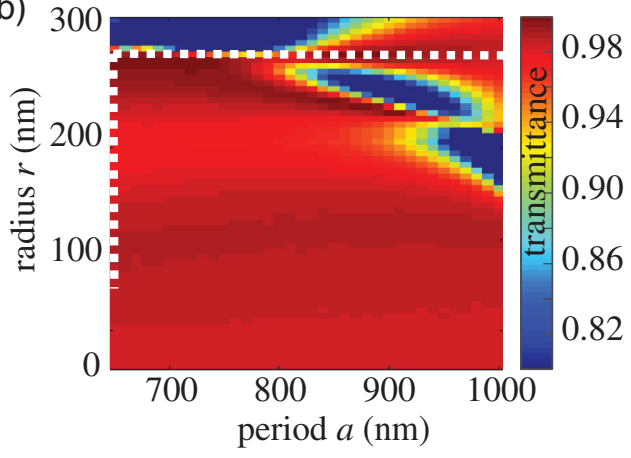

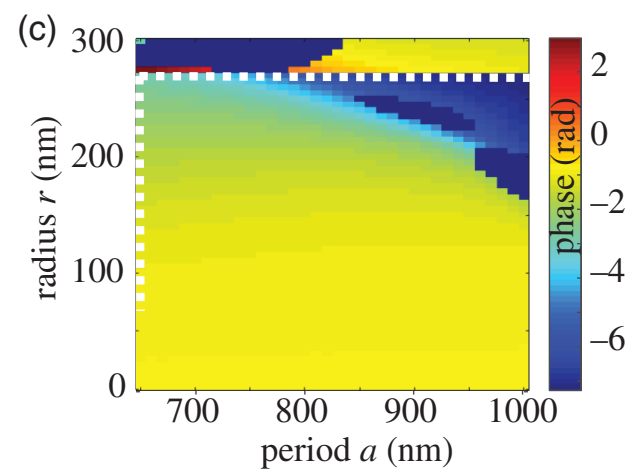
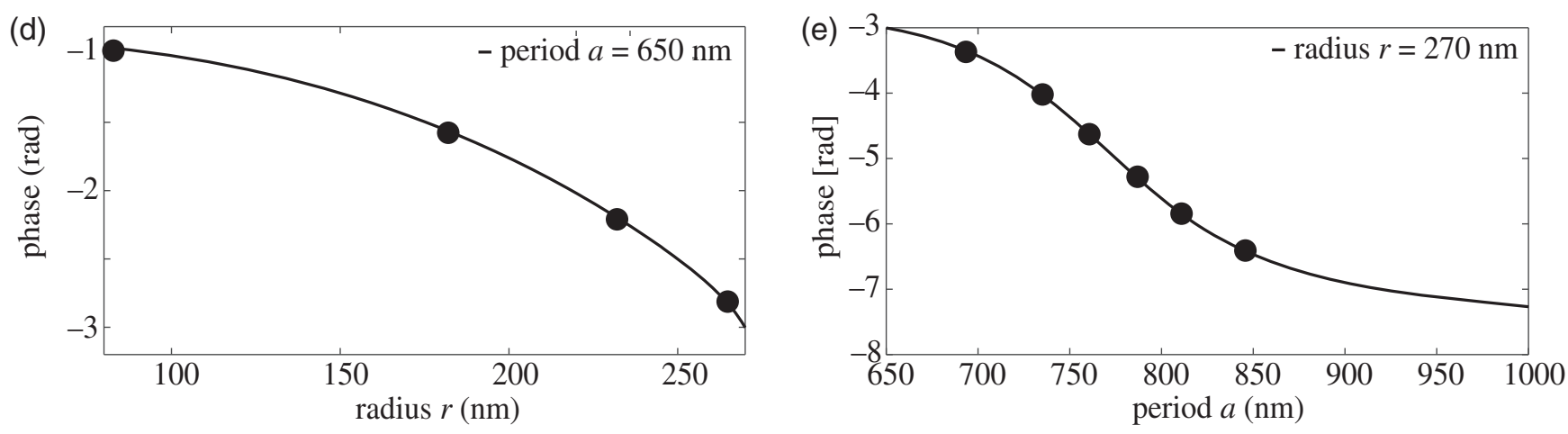

Fig. 3 (a) Sketch of the considered geometry of the metasurface consisting of silicon nanodisks embedded in a medium with a refractive index of $n=1.45$. Numerical calculations of (b) the transmittance intensity and (c) phase of silicon nanodisk arrays with varying radius and lattice periodicity. The operation wavelength is $1500 \mathrm{~nm}$. (d), (e) Transmission phase of metasurfaces with parameters along the white dashed lines in (b) and (c). 
geometric parameters disk height $h$, disk radius $r$, and period of the quadratic lattice $a$. The nanodisks are considered to be symmetrically embedded in a host material, which, in practice, will be achieved by using a substrate of $\mathrm{SiO}_{2}$ and by covering the disks after nanostructuring with spin-on-glass with the same refractive index $n=1.45$ as the substrate. To exactly fulfill the Huygens condition at a wavelength of $\lambda=1500 \mathrm{~nm}$, a geometry with $h=243 \mathrm{~nm}, r \approx 270 \mathrm{~nm}$, and $a \approx 840 \mathrm{~nm}$ is needed, ${ }^{12}$ where the period of the metasurface can be used as a parameter to control the phase. However, with this approach, the phase coverage is limited to $\frac{3}{2} \pi$. Hence, we use the disk radius as the second tunable geometry parameter to enable full-phase coverage with high transmission. ${ }^{46-48}$ In Figs. 3(b) and 3(c), we plot the simulated absolute value and phase change of light transmitted through such a periodic metasurface in dependence on period and disk radius. To focus on parameter regions with high transmission relevant for applications, the data are only shown for absolute values of the transmission larger than 0.8 . For the parameters along the dashed line in Figs. 3(b) and 3(c), the absolute value of transmission is always above 0.96 while the phase completely covers the range of $0-2 \pi$. To show this more clearly, we plot the achievable phase values in Figs. 3(d) and 3(e), where Fig. 3(d) shows the phases for a fixed period of $650 \mathrm{~nm}$ and varying radius and Fig. 3(e) shows the phases for a fixed radius of $270 \mathrm{~nm}$ and a varying period. The structure parameters to realize 10 evenly spaced phase steps are indicated by the dots in Figs. 3(d) and 3(e).

\section{Experimental Results}

Discretized phase masks of 1-cm diameter with 4, 6, 8, and 10 phase steps were fabricated based on commercially available amorphous silicon thin films on a glass substrate (Tafelmaier Dünnschicht Technik GmbH). The silicon layer was etched to the target height of $243 \mathrm{~nm}$ by argon-ion beam etching, covered with a chromium layer and a photoresist mask (100 nm EN038, Tokyo Ohka Kogyo Co., Ltd.), and exposed by a variable-shaped electron-beam lithography system (Vistec SB 350). The resist was developed for $30 \mathrm{~s}$ at room temperature in OPD 4262 and the pattern was transferred to the chromium layer by ion-beam etching (Oxford Ionfab 300). The silicon layer was then etched by reactive ion etching (RIE-ICP,
Sentech SI-500 C). After removal of the remaining resist (in acetone) and chromium (ceric ammonium nitrate-based solution), the sample was covered by spin-on-glass $(\sim 1 \mu \mathrm{m}$ Futurrex IC1-200) to ensure symmetric embedding of the nanostructures. Furthermore, the layer serves to reduce the index contrast between the nanodisks and their surroundings, resulting in electric and magnetic dipole resonances of similar spectral width, thus facilitating the formation of a broadband Huygens' regime. ${ }^{43}$ An SEM image of a portion of the sample before the embedding is shown in Fig. 4(a), where the individual nanostructures are clearly visible. The structures are etched deeper than just through the silicon film of 243-nm thickness; however, this does not influence their optical performance, due to the symmetric embedding. In Fig. 4(b), we show a photograph of the finished sample. The four different discretizations are visible in the four rows, and the different columns correspond to different exposure doses. These different doses lead to nanodisks of slightly different radii, such that the resulting phase masks have different operational wavelengths. Different regions of the phase mask can be identified by the different colors in the photograph. They result from the diffraction of the visible illumination light, which has a shorter wavelength than the periods of the metasurfaces.

To verify the phase profiles of the manufactured phase masks, we first measured the transmission phase for plane wave illumination using an imaging interferometer. ${ }^{6,12}$ Results for a phase mask with 10 phase levels and operation wavelength of $1490 \mathrm{~nm}$, close to the design wavelength, are shown in Fig. 4(c). Due to the limited field-of-view of the experimental setup used, only the central portion of the phase mask is depicted. However, it is clearly visible that 10 different phase levels with even spacing are realized in a spatial distribution corresponding to the design shown in Fig. 2(b). We note that the measured absolute phases are different than in the design, as our measurement setup provides no absolute reference. Furthermore, we characterized the transmission amplitude of the different regions within the phase mask at $1490 \mathrm{~nm}$. We found that for some of the regions, the transmission was lower than expected from the simulations, with the minimum value being about 0.5 , whereas others realize near unity transmission. This can be explained by slightly smaller radii of the realized structures with
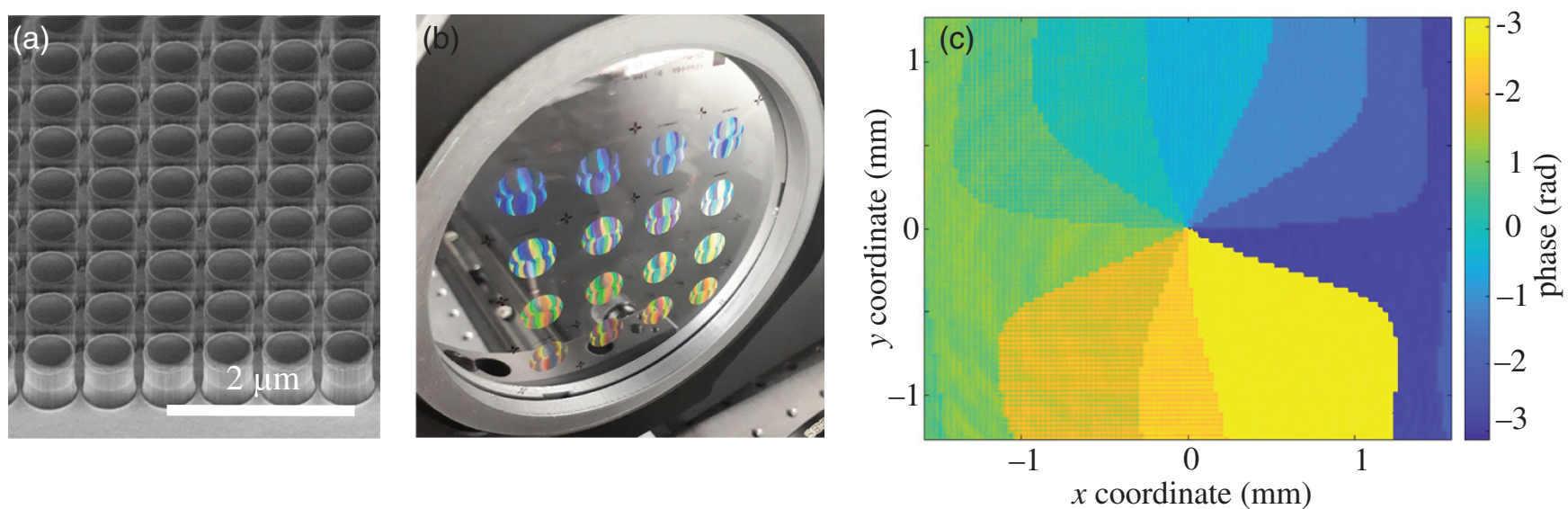

Fig. 4 (a) SEM image of realized silicon metasurface before covering with spin-on-glass. The scale bar is $2 \mu \mathrm{m}$. (b) Photograph of the finished sample with the 16 fabricated phase masks of $1-\mathrm{cm}$ diameter. (c) Measured phase profile in the center of a phase mask with 10 phase levels. 
respect to the design, leading to a violation of the perfect Huygens' condition for some regions. As can be seen in Figs. 3(b) and 3(c), already a slightly smaller radius leads to decreased transmission along the horizontal dashed line while the phases are not affected. The transmission of the phase mask for illumination with a point source as used in the experiment described below, which illuminates all regions, was above 0.7 .

To characterize the function of the realized phase masks, we used a setup similar to the sketch in Fig. 1(a). The illuminating laser light was emitted from a single-mode fiber. After $D \approx 60 \mathrm{~cm}$ propagation length between the fiber and the phase mask, the incident light approximates a spherical-wave illumination of the phase mask. Directly after the phase mask, a diaphragm is used to block all light not transmitted through the phase mask. A lens after the diaphragm then imaged the light on an indium gallium arsenide camera with $640 \times 512$ pixel resolution and $25-\mu \mathrm{m}$ pixel pitch. In contrast to the simulations and experimental results discussed so far, in this configuration also light with non-normal incidence passes through the phase mask in the imaging system. The transmission and phase of Huygens' metasurfaces can be strongly influenced already by moderately oblique incidence angles ${ }^{49}$ However, for the metasurface geometry and dimensions used in this work, it was demonstrated that the beam inclination angles of below 3 deg present in our imaging system will not severely change the properties of the phase mask. ${ }^{50}$
In Fig. 5(a), we show an example of a DH-PSF imaged with a lens of the focal length $f=250 \mathrm{~mm}$ measured for 1490-nm wavelength. Clearly, the two expected maxima are visible. The amount of light not localized in the two main lobes is comparable to numerically obtained DH-PSFs with the ideal phase mask, as shown in Fig. 2(c), underlining the negligible effect of non-normal incidence angles in our experiment. In the following experiments, we reduced the focal length to $f=200 \mathrm{~mm}$ to maximize the field-of-view of the 3-D imaging system. Decreasing the focal length even further would lead to a PSF peak separation of less than 10 image pixels, which deteriorates the depth reconstruction robustness using the approach shown in Ref. 37.

First, we analyzed the dependence of the focal intensity distribution on the distance $D$ between source and imaging system for all four different discretization levels of the samples operating at wavelengths around $1490 \mathrm{~nm}$. For all discretizations, two-lobed focal intensity distributions could be measured. In Fig. 5(b), we plot the angle $\Theta$ of these distributions in dependence on the distance $D$ and the discretization level. Clearly, the angle $\Theta$ changes upon change of the distance $D$, with a linear relationship between both parameters, which is independent of the phase discretization, as predicted by our simulations. Within a rotation of $180 \mathrm{deg}$, a change of the source distance $D$ of $190 \mathrm{~mm}$ can be uniquely identified. The accessible distance spans values from 540 to $730 \mathrm{~mm}$. The insets show two specific (a)

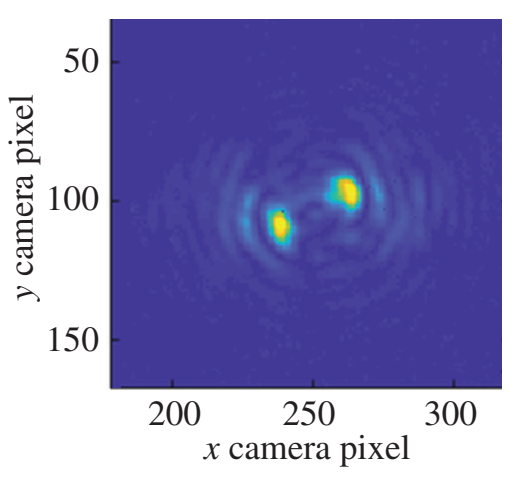

(b)

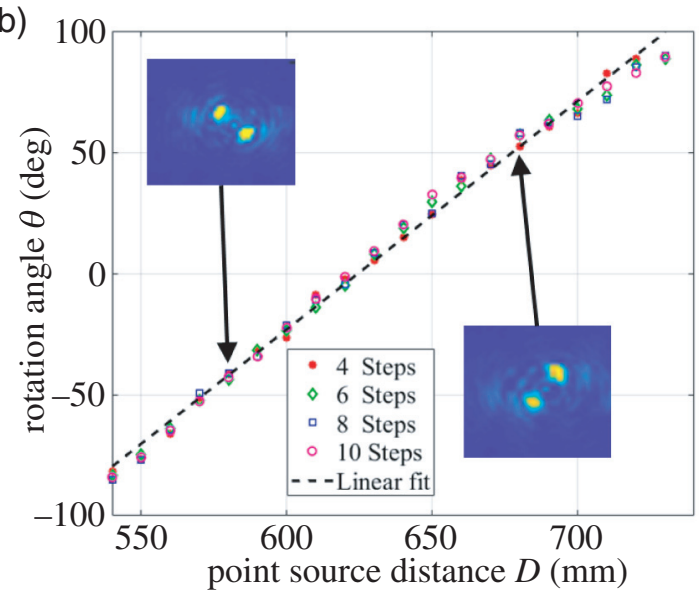

(c)

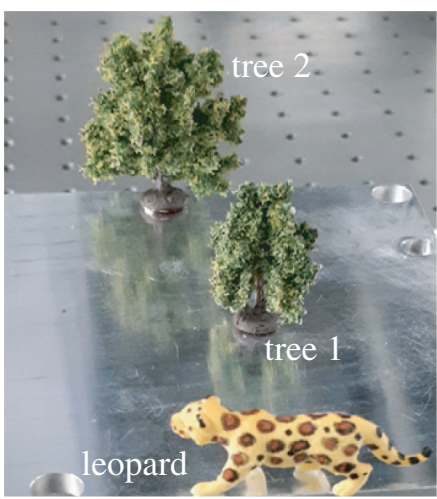

(d)

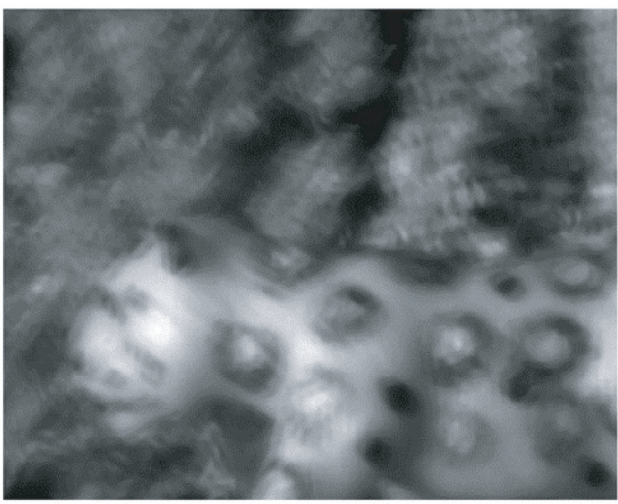

(e)

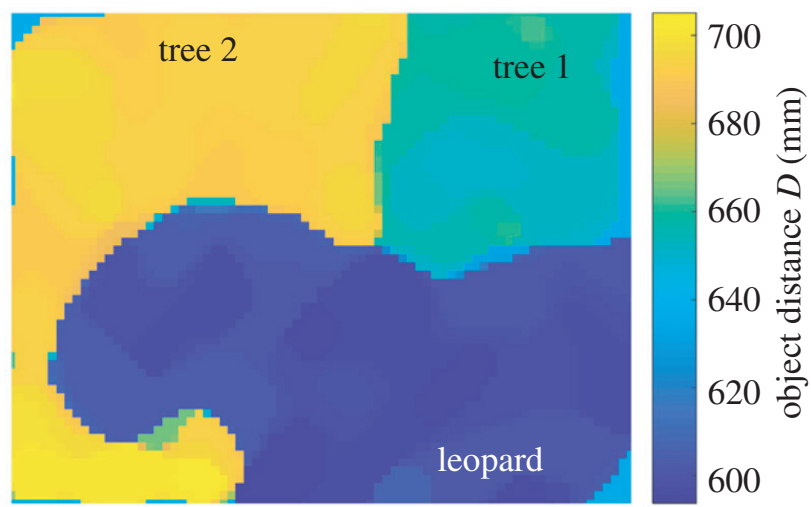

Fig. 5 (a) Measured DH-PSF. (b) Rotation angle of the DH-PSF in dependence on the sourcesample distance $D$, the insets show two specific orientations of the DH-PSF. (c) Photograph of a 3-D scene. (d) Raw image that was taken using the phase mask and (e) retrieved depth information. 
realizations of the DH-PSF as created by the phase mask with 10 phase levels. The distance between the two maxima is around 12 pixels; their FWHM is about 2 pixels. This is similar for all discretization levels, although the intensity distributed outside the two main maxima is growing for a decreasing number of phase levels. We note that the achievable depth resolution does not mainly depend on the width of the individual maxima but rather on the accuracy in determining their position and the rotation angle of their connecting line. Hence, the depth resolution limit is fundamentally given by the signal-to-noise ratio of the measurement, similar to fluorescence localization imaging, ${ }^{51}$ and could be quantified using the Cramer-Rao lower bound for a given photon number. ${ }^{34}$ We also investigated the dependence of the obtained focal distribution on the excitation laser wavelength. For the phase mask with 10 levels, the DH-PSF could still be obtained for wavelength differences of up to $10 \mathrm{~nm}$ between the exciting laser and the central operation wavelength without a wavelength-induced rotation but with increasing FWHM of the two maxima, which eventually merge. This limit of the operation bandwidth of $20 \mathrm{~nm}$ is due to the resonant nature of the used metasurfaces, which leads to a changing phase profile for changing wavelength. Thus, outside this bandwidth, a different PSF will be realized.

After the described characterization of the fundamental properties of the realized phase masks, we also demonstrate singlelens 3-D imaging. To this end, we adapt the experimental setup. The point source used up to now is replaced by a 3-D scene comprising from a model leopard and two model trees located at three different object distances, respectively. This scene is shown in Fig. 5(c) from a different viewing angle than in the measurement to visualize the 3-D arrangement of the three elements. The scene is illuminated by incoherent light from a halogen lamp to avoid the formation of laser speckles in the image. To restrict the bandwidth of the measured signal to the operation bandwidth of the phase mask, a bandpass filter of 12-nm FWHM and 1490-nm central wavelength is placed in front of the camera. In the measurement, the scene is observed from an angle such that all three objects appear behind each other. The raw image of the scene as taken by the camera is shown in Fig. 5(d). The image appears washed out due to the DH-PSF, which creates two shifted images from every object point superimposed incoherently across the image. From this data, the distance of the different object points can be retrieved by numerically analyzing the image cepstrum and retrieving the local orientation of the DH-PSF. ${ }^{37}$ This is done by analyzing smaller patches of the measured wide-field image individually. Here, a field of $128 \times 128$ pixels was analyzed to determine the local object distance $D$. By rastering this field of analysis across the measured image, the depth information shown in Fig. 5(e) was obtained. Clearly, the different distances of the three objects can be discriminated, demonstrating 3-D imaging using a metasurface-based optical system. The objects in the investigated scene were situated in distances of $\sim 700,660$, and $600 \mathrm{~mm}$, respectively, but the complete range of distances within one 180 deg rotation of the PSF as discussed above can, in principle, be accessed.

\section{Discussion and Conclusion}

As can be seen in the image, the resolution of the obtained depth map is lower than the $640 \times 512$ pixel resolution of the employed camera. In addition to the DH-PSF, which mixes object information from adjacent locations, this is due to the rather large patch size needed for a robust cepstrum analysis. For the limited resolution of the available camera, this leads to a rather small effective resolution of the depth map, especially visible for the small distance variations within the individual objects, which are hardly resolved. However, large differences in the distance between the objects can be distinguished with high spatial resolution, as evidenced by the sharp boundaries in Fig. 5(e). This is due to the fact that, in the case where different objects within the analyzed patch lead to PSF rotations larger apart than the FWHM of the individual PSF maxima, the image cepstrum is composed of two pairs of maxima. In the analysis, only the pair with a larger amplitude is used, leading to a sharp transition. The depth difference under which this high spatial resolution could be obtained is limited by the FWHM of the PSF maxima. For our experimental parameters, this corresponds to $\sim 10-\mathrm{mm}$ depth difference.

One constraint arises from the angle dependence of the used Huygens' metasurfaces, which limits the numerical aperture that can be used for a 3-D imaging system based on such phase masks. In our experimental implementation, the numerical aperture was about 0.02 , which is sufficient for imaging of distant objects. Larger values might be accessible if optimized imaging systems are used, e.g., telecentric imaging systems or multilens systems, where the phase mask may be placed in a plane with minimal incidence angles. Otherwise, nanostructures optimized for use with lenses with high numerical aperture ${ }^{7}$ may be employed to implement the phase mask.

Our results clearly demonstrate the suitability of metasurfaces for the implementation of complex optical elements for single-lens, polarization-insensitive 3-D imaging. As such metasurfaces can also be realized in the visible spectral range, where cameras based on silicon sensor chips with much higher resolution and lower noise are available, increased quality of the measurable depth maps is possible. Furthermore, more complicated rotating PSFs could be implemented to improve the robustness of the depth retrieval algorithm. ${ }^{38}$ Finally, the additional lens used in our experiment could be also implemented in the phase mask, leading to one complex, ultra-thin optical element for 3-D imaging.

These optimizations can all be implemented using the presented principal structure design. Additionally, using the strong dispersion of the metasurface also, enhanced functionalities could be implemented. For instance, different phase profiles can be implemented in one optical element for different wavelengths $s^{40}$ or polarizations. This could be used to create conventional lenses and elements for 3-D imaging in one metasurface, where the function can be selected by wavelength or polarization.

To conclude, here, we designed and implemented a Huygens'-metasurface-based phase mask that enables 3-D imaging using only one camera. With this, we took another step toward the use of metasurfaces not only to replace lenses in imaging optics but also to introduce more complicated functionalities into imaging systems.

\section{Acknowledgments}

We thank Waltraud Gräf, Daniel Voigt, Michael Banasch, and Thomas Käsebier for help with the fabrication. Financial support by the German Research Foundation within the Emmy-Noether program and the SPP "Tailored Disorder" 
(STA 1426/1-1, STA 1426/2-1, PE 1524/10-2) is gratefully acknowledged. Part of this research was funded by the German Federal Ministry of Education and Research (BMBF) under the project identifiers $13 \mathrm{~N} 14147$ and 03ZZ0434. Responsibility for the content of this work resides with the authors. C.J. acknowledges funding from the University of the Chinese Academy of Sciences' "International Cooperation Training Program for Doctoral Students, Fraunhofer Program.” The authors have no relevant financial interests in the manuscript and no other potential conflicts of interest to disclose.

\section{References}

1. N. Yu and F. Capasso, "Flat optics with designer metasurfaces," Nat. Mater. 13(2), 139-150 (2014).

2. M. Decker and I. Staude, "Resonant dielectric nanostructures: a low-loss platform for functional nanophotonics," J. Opt. 18(10), 103001 (2016).

3. P. Genevet et al., "Recent advances in planar optics: from plasmonic to dielectric metasurfaces," Optica 4(1), 139-152 (2017).

4. S. M. Kamali et al., "A review of dielectric optical metasurfaces for wavefront control," Nanophotonics 7(6), 1041-1068 (2018).

5. N. Meinzer, W. L. Barnes, and I. R. Hooper, "Plasmonic meta-atoms and metasurfaces," Nat. Photonics 8(12), 889-898 (2014).

6. K. E. Chong et al., "Efficient polarization-insensitive complex wavefront control using Huygens? Metasurfaces based on dielectric resonant meta-atoms," ACS Photonics 3(4), 514-519 (2016).

7. M. Khorasaninejad et al., "Metalenses at visible wavelengths: diffraction-limited focusing and subwavelength resolution imaging," Science 352(6290), 1190-1194 (2016).

8. L. Wang et al., "Grayscale transparent metasurface holograms," Optica 3(12), 1504-1505 (2016).

9. B. Wang et al., "Visible-frequency dielectric metasurfaces for multiwavelength achromatic and highly dispersive holograms," Nano Lett. 16(8), 5235-5240 (2016).

10. L. Jin et al., "Noninterleaved metasurface for $\left(2^{6}-1\right)$ spin-and wavelength-encoded holograms," Nano Lett. 18(12), 8016-8024 (2018).

11. Y. Yang et al., "Dielectric meta-reflectarray for broadband linear polarization conversion and optical vortex generation," Nano Lett. 14(3), 1394-1399 (2014).

12. K. E. Chong et al., "Polarization-independent silicon metadevices for efficient optical wavefront control," Nano Lett. 15(8), 53695374 (2015).

13. T. Stav et al., "Quantum entanglement of the spin and orbital angular momentum of photons using metamaterials," Science 361(6407), 1101-1104 (2018).

14. M. Khorasaninejad et al., "Achromatic metasurface lens at telecommunication wavelengths," Nano Lett. 15(8), 5358-5362 (2015).

15. A. Arbabi et al., "Efficient dielectric metasurface collimating lenses for mid-infrared quantum cascade lasers," Opt. Express 23(26), 33310-33317 (2015).

16. E. Arbabi et al., "Multiwavelength polarization-insensitive lenses based on dielectric metasurfaces with meta-molecules," Optica 3(6), 628-633 (2016).

17. E. Arbabi et al., "High efficiency double-wavelength dielectric metasurface lenses with dichroic birefringent meta-atoms," Opt. Express 24(16), 18468-18477 (2016).

18. A. Zhan et al., "Low-contrast dielectric metasurface optics," ACS Photonics 3(2), 209-214 (2016).

19. M. P. Backlund et al., "Removing orientation-induced localization biases in single-molecule microscopy using a broadband metasurface mask," Nat. Photonics 10(7), 459-462 (2016).
20. H. Zuo et al., "High-efficiency all-dielectric metalenses for midinfrared imaging," Adv. Opt. Mater. 5(23), 1700585 (2017).

21. M. Khorasaninejad and F. Capasso, "Metalenses: versatile multifunctional photonic components," Science 358(6367), eaam8100 (2017).

22. M. M. Shanei et al., "Dielectric metalenses with engineered point spread function," Appl. Opt. 56(32), 8917-8923 (2017).

23. R. Paniagua-Dominguez et al., "A metalens with a near-unity numerical aperture," Nano Lett. 18(3), 2124-2132 (2018).

24. S. M. Kamali et al., "Decoupling optical function and geometrical form using conformal flexible dielectric metasurfaces," Nat. Commun. 7, 11618 (2016).

25. S. M. Kamali et al., "Highly tunable elastic dielectric metasurface lenses," Laser Photonics Rev. 10(6), 1002-1008 (2016).

26. E. Arbabi et al., "MEMS-tunable dielectric metasurface lens," Nat. Commun. 9(1), 812 (2018).

27. B. Cyganek and J. P. Siebert, An Introduction to $3 D$ Computer Vision Techniques and Algorithms, John Wiley \& Sons, Chippenham (2011).

28. J. Geng, "Structured-light 3D surface imaging: a tutorial," $A d v$. Opt. Photonics 3(2), 128-160 (2011).

29. H. Kwon et al., "Computational complex optical field imaging using a designed metasurface diffuser," Optica 5(8), 924-931 (2018).

30. Z. Yang et al., "Generalized Hartmann-Shack array of dielectric metalens sub-arrays for polarimetric beam profiling," Nat. Commun. 9(1), 4607 (2018).

31. R. J. Lin et al., "Achromatic metalens array for full-colour lightfield imaging," Nat. Nanotechnol. 14(3), 227-231 (2019).

32. A. Greengard, Y. Y. Schechner, and R. Piestun, "Depth from diffracted rotation," Opt. Lett. 31(2), 181-183 (2006).

33. S. Quirin and R. Piestun, "Depth estimation and image recovery using broadband, incoherent illumination with engineered point spread functions," Appl. Opt. 52(1), A367-A376 (2013).

34. S. R. P. Pavani, A. Greengard, and R. Piestun, "Three-dimensional localization with nanometer accuracy using a detector-limited double-helix point spread function system," Appl. Phys. Lett. 95(2), 021103 (2009)

35. S. R. P. Pavani, J. G. DeLuca, and R. Piestun, "Polarization sensitive, three-dimensional, single-molecule imaging of cells with a double-helix system," Opt. Express 17(22), 19644-19655 (2009).

36. S. R. P. Pavani et al., "Three-dimensional, single-molecule fluorescence imaging beyond the diffraction limit by using a doublehelix point spread function," Proc. Natl. Acad. Sci. U.S.A. 106(9), 2995-2999 (2009).

37. R. Berlich, A. Bräuer, and S. Stallinga, "Single shot three-dimensional imaging using an engineered point spread function," Opt. Express 24(6), 5946-5960 (2016).

38. R. Berlich and S. Stallinga, "High-order-helix point spread functions for monocular three-dimensional imaging with superior aberration robustness," Opt. Express 26(4), 4873-4891 (2018).

39. S. Jeon et al., "Three-dimensional nanofabrication with rubber stamps and conformable photomasks," Adv. Mater. 16(15), 13691373 (2004).

40. B. Walther et al., "Spatial and spectral light shaping with metamaterials," Adv. Mater. 24(47), 6300-6304 (2012).

41. M. Khorasaninejad et al., "Multispectral chiral imaging with a metalens," Nano Lett. 16(7), 4595-4600 (2016).

42. K. Wang et al., "Quantum metasurface for multiphoton interference and state reconstruction," Science 361(6407), 1104-1108 (2018).

43. M. Decker et al., "High-efficiency dielectric Huygens surfaces," Adv. Opt. Mater. 3(6), 813-820 (2015).

44. Z. Dong et al., "Printing beyond sRGB color gamut by mimicking silicon nanostructures in free-space," Nano Lett. 17(12), 76207628 (2017).

45. A. Arbabi et al., "Dielectric metasurfaces for complete control of phase and polarization with subwavelength spatial resolution and high transmission," Nat. Nanotechnol. 10(11), 937-943 (2015). 
46. M. I. Shalaev et al., "High-efficiency all-dielectric metasurfaces for ultracompact beam manipulation in transmission mode," Nano Lett. 15(9), 6261-6266 (2015).

47. Y. F. Yu et al., "High-transmission dielectric metasurface with $2 \pi$ phase control at visible wavelengths," Laser Photonics Rev. 9(4), 412-418 (2015).

48. A. J. Ollanik et al., "High-efficiency all-dielectric Huygens metasurfaces from the ultraviolet to the infrared," ACS Photonics 5(4), 1351-1358 (2018).

49. P. Lalanne and P. Chavel, "Metalenses at visible wavelengths: past, present, perspectives," Laser Photonics Rev. 11(3), 1600295 (2017).

50. D. Arslan et al., "Angle-selective all-dielectric Huygens' metasurfaces," J. Phys. D Appl. Phys. 50(43), 434002 (2017).

51. E. Betzig et al., "Imaging intracellular fluorescent proteins at nanometer resolution," Science 313(5793), 1642-1645 (2006).
Chunqi Jin is a doctoral candidate at the University of the Chinese Academy of Sciences. She had a one-year cooperation training at Friedrich-Schiller-Universität Jena. Her research interests consist of metasurface and its applications in three-dimensional imaging and sensing.

Frank Setzpfandt obtained his $\mathrm{PhD}$ from Friedrich-Schiller-Universität Jena in 2012. After a post-doc with the Australian National University he returned to Jena, where he is currently leading a junior research group at the Institute of Applied Physics. His research interests comprise nonlinear and quantum optics in waveguides and nanostructures, as well as classical and quantum imaging.

Biographies of the other authors are not available. 